\title{
Radiotherapy with Intensity-Modulated (IMRT) Techniques in the Treatment of Anal Carcinoma (RAINSTORM): A Multicenter Study on Behalf of AIRO (Italian Association of Radiotherapy and Clinical Oncology) Gastrointestinal Study Group
}

Luciana Caravatta $^{1, *\left(\mathbb{D}, \text { Giovanna Mantello }^{2}\left(\mathbb{D}, \text { Francesca Valvo }^{3} \text {, Pierfrancesco Franco }\right.\right.}{ }^{4} \mathbb{D}$, Lucrezia Gasparini ${ }^{1}$, Consuelo Rosa ${ }^{1}$, Najla Slim ${ }^{5}$, Stefania Manfrida ${ }^{6}{ }^{\circ}$, Francesca De Felice ${ }^{7}$, Marianna A. Gerardi ${ }^{8}$,

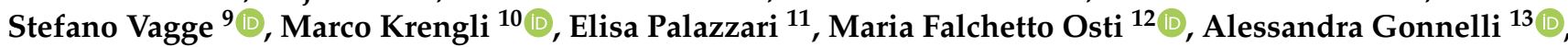
Gianpiero Catalano ${ }^{14}{ }^{-}$, Patrizia Pittoni ${ }^{15}$, Giovani Battista Ivaldi ${ }^{16}{ }^{-1}$, Alessandra Galardi ${ }^{17}$, Marco Lupattelli ${ }^{18}$, Maria Elena Rosetto ${ }^{19}$, Rita Marina Niespolo ${ }^{20}$, Alessandra Guido ${ }^{21}$, Oreste Durante ${ }^{22}\left(\right.$, Gabriella Macchia ${ }^{23}(\mathbb{D}$, Fernando Munoz ${ }^{24}{ }^{\circledR}$, Badr El khouzai ${ }^{25}$, Maria Rosaria Lucido ${ }^{26}$, Annamaria Porreca ${ }^{27} \mathbb{C}$, Marta Di Nicola ${ }^{27}(\mathbb{D}$, Maria Antonietta Gambacorta ${ }^{6}$, Vittorio Donato ${ }^{28,+}$ and Domenico Genovesi ${ }^{1,29,+}$

check for updates

Citation: Caravatta, L.; Mantello, G.; Valvo, F.; Franco, P.; Gasparini, L.; Rosa, C.; Slim, N.; Manfrida, S.; De Felice, F.; Gerardi, M.A.; et al.

Radiotherapy with

Intensity-Modulated (IMRT)

Techniques in the Treatment of Anal Carcinoma (RAINSTORM): A

Multicenter Study on Behalf of AIRO (Italian Association of Radiotherapy and Clinical Oncology)

Gastrointestinal Study Group. Cancers 2021, 13, 1902. https://doi.org/

10.3390/cancers 13081902

Academic Editor: Oliver J. Ott

Received: 25 February 2021

Accepted: 12 April 2021

Published: 15 April 2021

Publisher's Note: MDPI stays neutral with regard to jurisdictional claims in published maps and institutional affiliations.

Copyright: (C) 2021 by the authors Licensee MDPI, Basel, Switzerland. This article is an open access article distributed under the terms and conditions of the Creative Commons Attribution (CC BY) license (https:/ / creativecommons.org/licenses/by/ $4.0 /)$.
1 Radiation Oncology Unit, “SS Annunziata” Hospital, “G. D'Annunzio” University of Chieti-Pescara, 66100 Chieti, Italy; luky.gasp@gmail.com (L.G.); consuelo.rosa@unich.it (C.R.); d.genovesi@unich.it (D.G.)

2 Department of Oncology and Radiotherapy, Azienda Ospedaliero Universitaria Ospedali Riuniti, 60002 Ancona, Italy; gio@mobilia.it

3 Radiotherapy Unit, Clinical Department, CNAO National Center for Oncological Hadrontherapy, 27100 Pavia, Italy; francesca.valvo@cnao.it

4 Department of Oncology, Radiation Oncology, University of Turin, 10126 Turin, Italy; pierfrancesco.franco@unito.it

5 Department of Radiotherapy, IRCCS San Raffaele Scientific Institute, 20132 Milan, Italy; slim.najla@hsr.it

6 "A. Gemelli" IRCCS, UOC di Radioterapia Oncologica, Dipartimento di Diagnostica per Immagini, Radioterapia Oncologica ed Ematologia, Fondazione Policlinico Universitario, 00168 Rome, Italy; stefania.manfrida@policlinicogemelli.it (S.M.); mariaantonietta.gambacorta@policlinicogemelli.it (M.A.G.)

7 Department of Radiotherapy, Policlinico Umberto I, "Sapienza” University of Rome, 00168 Rome, Italy; francesca.defelice@uniroma1.it

8 Department of Radiotherapy, IEO European Institute of Oncology, IRCCS, 20121 Milan, Italy; marianna.gerardi@ieo.it

9 Department of Radiation Oncology, IRCCS Ospedale Policlinico San Martino, 16132 Genoa, Italy; stefano.vagge@hsanmartino.it

10 Division of Radiation Oncology, Department of Translational Medicine, University Hospital "Maggiore della Carità", University of Piemonte Orientale, 28100 Novara, Italy; marco.krengli@med.uniupo.it

11 Oncological Referral Center, Radiation Oncology Department, 33081 Aviano, Italy; elisa.palazzari@cro.it

12 Unit of Radiation Oncology, Sant'Andrea Hospital, Sapienza University of Rome, 00189 Rome, Italy; mattiafalchetto.osti@uniroma1.it

13 Department of Radiotherapy, Azienda Ospedaliero-Universitaria Pisana, 56124 Pisa, Italy; alessandra.gonnelli@phd.unipi.it

14 Radiation Oncology Center, IRCCS Multimedica, Sesto San Giovanni, 20099 Milan, Italy; gianpiero.catalano@multimedica.it

15 Radiation Oncology Unit, Asst Lariana, Ospedale di Como, 22100 Como, Italy; patrizia.pittoni@asst-lariana.it

16 Radiation Oncology Unit, ICS Maugeri, IRCCS, 27100 Pavia, Italy; giovannibattista.ivaldi@icsmaugeri.it

17 Department of Radiotherapy, University Hospital, 50134 Florence, Italy; galardia@aou-careggi.toscana.it

18 Radiation Oncology Section, University of Perugia and Perugia General Hospital, 06156 Perugia, Italy; mlupattelli62@gmail.com

19 Radiotherapy Unit, Belcolle Hospital, 01100 Viterbo, Italy; mariaelenarosetto@virgilio.it

20 Radiotherapy Unit, Azienda Ospedaliera San Gerardo, 20900 Monza, Italy; ritamarinaniespolo@gmail.com

21 Radiation Oncology, IRCCS Azienda Ospedaliero-Universitaria di Bologna, 40138 Bologna, Italy; alessandraguido2008@gmail.com

22 Azienda Ospedaliera SS, Antonio e Biagio e Cesare Arrigo, 15121 Alessandria, Italy; oreste.durante@ospedale.al.it

23 Radiation Oncology Unit, Gemelli Molise Hospital-Università Cattolica del Sacro Cuore, 86100 Campobasso, Italy; gabriella.macchia@gemellimolise.it

24 Department of Radiotherapy, Azienda U. S. L. della Valle d'Aosta, 11100 Aosta, Italy; fmunoz@ausl.vda.it

25 Radiotherapy and Nuclear Medicine Unit, Veneto Institute of Oncology-IRCCS, 35128 Padova, Italy; badr.elkhouzai@iov.veneto.it 
26 Radiotherapy Unit, Ospedale Sanremo-ASL 1 Imperiese, 18038 Sanremo, Italy; m.lucido@asl1.liguria.it

27 Laboratory of Biostatistics, Department of Medical, Oral and Biotechnological Sciences, "G. D'Annunzio" University of Chieti-Pescara, 66100 Chieti, Italy; annamaria.porreca@unich.it (A.P.); marta.dinicola@unich.it (M.D.N.)

28 Radiation Oncology, Azienda Ospedaliera San Camillo-Forlanini, 00152 Rome, Italy; vittoriodonato@libero.it

29 Department of Neuroscience, Imaging and Clinical Sciences, “G. D'Annunzio" University of Chieti-Pescara, 66100 Chieti, Italy

* Correspondence: lcaravatta@hotmail.com or luciana.caravatta@asl2abruzzo.it; Tel.: +39-0871-358244; Fax: +39-0871-357473

+ Co-last author: Vittorio Donato \& Domenico Genovesi.

Simple Summary: Concurrent chemo-radiotherapy is the standard treatment in anal cancer. Intensity-modulated radiotherapy (IMRT) was proved to reduce severe, acute and late toxicities. Moreover, IMRT techniques allow for the planning and delivery of a simultaneous integrated boost (SIB), with a differential dose per fraction given to selected sub-regions during the same treatment session. This boost modality provides the chance to employ a dose-painted approach with a reduction in overall treatment time that could result in a potential clinical advantage. Since a large variability in dose prescription to the primary tumor and elective or involved lymph nodes can be found in available guidelines and clinical practice, a multicenter analysis was conducted to evaluate the pattern of care and the impact of radiotherapy parameters on clinical outcomes for anal cancer patients treated with IMRT techniques within a national cohort.

Abstract: A multi-institutional retrospective study was conducted to evaluate the pattern of care and clinical outcomes of anal cancer patients treated with intensity-modulated radiotherapy (IMRT) techniques. In a cohort of 987 patients, the clinical complete response (CR) rate (beyond 6 months) was 90.6\%. The 3-year local control (LC) rate was 85.8\% (95\% CI: 84.4-87.2), and the 3-year colostomyfree survival (CFS) rate was 77.9\% (95\% CI: 76.1-79.8). Three-year progression-free survival (PFS) and overall survival (OS) rates were $80.2 \%$ and $88.1 \%$ (95\% CI: 78.8-89.4) (95\% CI: 78.5-81.9), respectively. Histological grade 3 and nodal involvement were associated with lower CR ( $p=0.030$ and $p=0.004$, respectively). A statistically significant association was found between advanced stage and nodal involvement, and LC, CFS, PFS, OS and event-free survival (EFS). Overall treatment time (OTT) $\geq 45$ days showed a trend for a lower PFS ( $p=0.050$ ) and was significantly associated with lower EFS $(p=0.030)$ and histological grade 3 with a lower LC $(p=0.025)$. No statistically significant association was found between total dose, dose/fraction and/or boost modality and clinical outcomes. This analysis reports excellent clinical results and a mild toxicity profile, confirming IMRT techniques as standard of care for the curative treatment of anal cancer patients. Lymph node involvement and histological grade have been confirmed as the most important negative prognostic factors.

Keywords: anal carcinoma; concomitant radio-chemotherapy; intensity-modulated radiotherapy; simultaneous integrated boost

\section{Introduction}

The primary aim of curative chemo-radiotherapy (CRT) for stage II-III anal cancer (AC) patients is to achieve locoregional control, while preserving the anal sphincter with intact function and avoiding a colostomy, with a reasonable quality of life. Since definitive CRT showed a high rate of clinical complete response (cCR up 80-90\%) and local control (LC = 70-60\% at 3 years) in several randomized studies [1-9], it currently represents the mainstay of treatment in this clinical setting. However, curative CRT is reported to be associated with a considerable rate of acute toxicities that could require treatment breaks with a prolonged overall treatment time (OTT), potentially affecting clinical outcomes $[10,11]$. Late effects could also be relevant. Highly conformal radiation modalities, such as intensity-modulated radiation therapy (IMRT), may reduce radiation dose to normal tissue with decreased toxicity and similar locoregional control rates [12,13]. In a large retrospective analysis of 
151 patients with AC treated with IMRT, an LC rate of $87 \%$ at 3 years was reported with low grade $\geq 3$ acute gastrointestinal (GI) (11\%) and skin (20\%) toxicities [14]. Moreover, IMRT offers the possibility to deliver a simultaneous integrated boost (SIB), with the chance to give different doses to treatment volumes in the same number of fractions, allowing a safe administration of higher doses to the gross tumor volume (GTV) and with a reduced OTT. Concerning this strategy, the RTOG 0529 trial evaluated dose-painted SIB-IMRT, reporting a significant reduction in $\geq \mathrm{G} 2$ acute hematologic, cutaneous and GI toxicity [15]. Several studies have confirmed a benefit in terms of oncological outcomes, toxicity rates and quality of life using IMRT techniques [16-22]. Based on these results, IMRT planning and delivery represent the standard radiotherapy (RT) option for AC, despite a lack of standardization concerning treatment volumes and doses, especially in the case of an SIB strategy. This variability could probably have an impact on treatment-related toxicity and long-term outcomes. Based on these considerations, a multi-institutional retrospective study was conducted with the aim to evaluate the clinical outcomes, patterns of care and the impact of RT parameters for AC patients treated with different IMRT techniques.

\section{Results}

\subsection{Patients and Treatment Characteristics}

A cohort of 987 consecutive non-metastatic AC patients treated between 2007-2019 was analyzed. Patients were enrolled within 25 different Italian centers. Clinical characteristics for all treated patients are shown in Table 1.

Table 1. Patient and tumor characteristics.

\begin{tabular}{|c|c|c|c|}
\hline \multicolumn{2}{|c|}{ Patient and Tumor Characteristics } & $n=987$ & $\%$ \\
\hline \multicolumn{4}{|c|}{ Age $=$ median 68.5 years (range: $54.00-80.00$ ) } \\
\hline \multirow{2}{*}{ Gender } & Male & 281 & 28.4 \\
\hline & Female & 706 & 71.5 \\
\hline \multirow{3}{*}{ ECOG Performance Status } & 0 & 775 & 78.5 \\
\hline & 1 & 197 & 19.9 \\
\hline & 2 & 15 & 1.5 \\
\hline \multirow{3}{*}{$\mathrm{HPV}$} & Negative & 210 & 21.3 \\
\hline & Positive & 229 & 23.2 \\
\hline & NR & 548 & 55.5 \\
\hline \multirow{3}{*}{ HIV } & Negative & 747 & 75.7 \\
\hline & Positive & 90 & 9.1 \\
\hline & NR & 150 & 15.2 \\
\hline \multirow{2}{*}{ Tumor site } & Anal Canal & 879 & 89.1 \\
\hline & Anal Margin & 108 & 10.9 \\
\hline \multirow{3}{*}{ Histology } & Squamous & 880 & 89.1 \\
\hline & Basalioid & 84 & 8.5 \\
\hline & Other & 23 & 2.3 \\
\hline \multirow{4}{*}{ Grading } & G1 & 68 & 6.9 \\
\hline & G2 & 328 & 33.2 \\
\hline & G3 & 233 & 23.6 \\
\hline & NR & 358 & 36.3 \\
\hline \multirow{3}{*}{ TNM Stage } & T1-T2, N0 & 330 & 33.4 \\
\hline & T3-T4, N0 & 106 & 10.7 \\
\hline & Any $T, N+$ & 551 & 55.8 \\
\hline \multirow{3}{*}{ Disease extension } & Early stage & 330 & 33.4 \\
\hline & LAD & 633 & 64.1 \\
\hline & ED & 24 & 2.4 \\
\hline
\end{tabular}

Legend: ECOG = Eastern Cooperative Oncology Group; HPV = human papilloma virus; HIV = human immunodeficiency virus; NR = non-reported; early disease: T1-T2 tumors; LAD = locally advanced disease (T3-T4 or $\mathrm{N}+$ = node-positive tumors); $\mathrm{ED}=$ extended disease (lumbar-aortic and/or common iliac lymph nodes involvement). 
An Eastern Cooperative Oncology Group (ECOG) performance status (PS) of 0 was reported in 775 patients $(78.5 \%)$. HIV positivity was identified in $90(9.1 \%)$ of the evaluated patients. In most of the patients, HPV status was not reported (55.5\%). Tumors were located within the anal canal in 879 patients $(89.1 \%)$. Basaloid histology was described in 84 patients $(8.5 \%)$.

Three hundred thirty patients (33.4\%) had cT1-2N0 stage, 106 patients $(10.6 \%)$ had cT3-4N0 stage and 551 (55.8\%) had lymph node involvement. Overall, 633 patients $(64.1 \%)$ presented with locally advanced disease (LAD) at diagnosis. Twenty-four patients (2.4\%) had a single site of clinical abdominal lymph node involvement (lumbar-aortic and/or common iliac lymph nodes).

Imaging modalities used to define tumor $(\mathrm{T})$, nodal $(\mathrm{N})$ and distant metastases $(\mathrm{M})$ stages are shown in Table 2.

Table 2. Imaging modalities used to define tumor $(\mathrm{T})$, nodal $(\mathrm{N})$ and distant metastases $(\mathrm{M})$ stages.

\begin{tabular}{ccccc}
\hline \multicolumn{1}{c}{ Staging } & \multicolumn{3}{c}{$\mathbf{N = 9 8 7}(\mathbf{9})$} \\
\hline \multirow{2}{*}{ Single Imaging modality } & & $\mathbf{T}$ & $\mathbf{N}$ & $\mathbf{M}$ \\
& CT & $143(14.5)$ & $186(18.8)$ & $402(40.7)$ \\
& MRI & $541(54.818)$ & $321(32.5)$ & $12(1.2)$ \\
& ERUS & $126(12.8)$ & $24(2.4)$ & $14(1.4)$ \\
Multiple Imaging modalities * & & $99(10.0)$ & $375(38)$ & $467(47.3)$ \\
\hline Missing data & & $78(7.9)$ & $79(8.2)$ & $81(8.2)$ \\
\hline
\end{tabular}

Legend: CT = computed tomography scan; MRI = magnetic resonance imaging; ERUS = endorectal ultrasound 18F-fluorodeoxyglucose positron emission tomography (18FDG-PET); *: all used diagnostic imaging in combinations.

Magnetic resonance imaging (MRI) was used in 541 patients (54.8\%) to define T stage; 18FDG-PET was used in 375 patients (38.0\%) and 467 patients (47.3\%) for $\mathrm{N}$ and $\mathrm{M}$ stage identification, respectively.

Treatment characteristics are detailed in Table 3.

Table 3. Treatment details.

\begin{tabular}{cccc}
\hline \multicolumn{2}{c}{ Treatment Details } & $\mathbf{n = 9 8 7}$ & \% \\
\hline Median Total Dose 55 Gy (range: 45-75) & & 30.6 \\
\hline \multirow{3}{*}{ IMRT modalities } & static or dynamic IMRT & 470 & 47.6 \\
& VMAT & 215 & 21.8 \\
\hline \multirow{5}{*}{ Boost modalities } & HT & 252 & 25.5 \\
& Sequential & 568 & 57.5 \\
& SIB & 122 & 12.4 \\
& SIB+ Sequential & 45 & 4.6 \\
\hline & SIB+ Sequential BRT & 634 & 64.2 \\
Concomitant & MMC + 5-FU & 145 & 14.7 \\
chemotherapy & MMC + Capecitabine & 21 & 2.1 \\
& CDDP + 5-FU & 67 & 6.8 \\
& CDDP + Capecitabine & 2 & 0.2 \\
& MMC & 8 & 0.8 \\
& 5-FU & 3 & 0.3 \\
& CDDP & 29 & 2.9 \\
& Capecitabine & 21 & 2.1 \\
& Other & 4 & 0.4 \\
& NR & 53 & 5.4
\end{tabular}

Legend: IMRT = intensity modulated radiation therapy; VMAT = volumetric modulated arc therapy; HT = helical tomotherapy; $\mathrm{SIB}=$ simultaneous integrated boost; $\mathrm{MMC}=$ mitomycin C; 5-FU = 5-fluorouracil; CDDP = cisplatin; $\mathrm{RT}=$ radiotherapy; $\mathrm{NR}=$ not reported . 
Three hundred and two patients (30.6\%) were treated with static or dynamic IMRT, 470 patients $(47.6 \%)$ with volumetric modulated arc therapy (VMAT) and 215 patients $(21.8 \%)$ with tomotherapy. A SIB strategy was used in 568 patients $(57.5 \%)$. An additional sequential boost was administered in 167 patients (16.9\%) using external beam RT (EBRT) or brachytherapy (BRT) in 122 patients (12.4\%) and 45 patients (4.6\%), respectively. Concomitant chemotherapy was administered in 934 patients $(94.63 \%)$. The mitomycin (MMC) plus 5-fluorouracil (5-FU) or capecitabine regimen was administered in 779 patients $(78.92 \%)$.

The total RT dose and daily fractionation prescription were analyzed according to clinical stage at presentation and reported based on the low-, intermediate- and high-risk planning target volumes (PTVs), as defined in the RTOG 0529 study [15]. The median total dose was 55 Gy (range: 45-75).

In patients with cT1-2 N0 disease, the elective low-risk PTV received a median dose of 45 Gy (range 32.40-48 Gy, 1.40-2 Gy daily) and high-risk PTV received a median dose of 54 Gy (range 37.50-70.40 Gy, 1.80-2.40 Gy daily), with a median total dose of 55 Gy (range 46-70.40 Gy).

In patients with cT3-T4 N0 disease, the elective low-risk PTV received a median dose of 45 Gy (range 34.50-48 Gy, 1.40-1.80 Gy daily) and high-risk PTV received a median dose of 54 Gy (range 41.40-68.40 Gy, 1.80-2.40 Gy daily), with a median total dose of 55 Gy (range 50-73 Gy).

In patients with cT1-T2 $\mathrm{N}+$ disease, the elective low-risk PTV received a median dose of 45 Gy (range 35.8-54 Gy, 1.40-2 Gy daily) and high-risk PTV received a median dose of 54 Gy (range 40-68.4 Gy, 1.80-2.40 Gy daily), with a median total dose of 55 Gy (range 45-74.4 Gy).

In patients with cT3-T4 $\mathrm{N}+$ disease, the elective low-risk PTV received a median dose of 45 Gy (range 36-54 Gy, 1.40-2 Gy daily) and high-risk PTV received a median dose of 55 Gy (range 44-72.4 Gy, 1.80-2.40 Gy daily), with a median total dose of 56 Gy (range 45-74.8 Gy).

\subsection{Treatment Compliance and Toxicity}

The median OTT was 45 days (range: 25-115). A treatment interruption of $>5$ days for toxicity occurred in 186 patients $(18.8 \%)$. RT was not definitively completed in 67 patients $(6.8 \%)$. Detailed toxicity profiles are shown in Table 4.

Table 4. Acute and late toxicities retrospectively graded according to Common Terminology Criteria for Adverse Events Version $4.0(n=987)$.

\begin{tabular}{|c|c|c|c|c|c|c|}
\hline \multicolumn{7}{|c|}{$n=987(\%)$} \\
\hline Grade & G0 & G1 & G2 & G3 & G4 & G3 and G4 \\
\hline \multicolumn{7}{|l|}{ ACUTE TOXICITY } \\
\hline Skin & $107(10.8)$ & $136(13.8)$ & $491(49.7)$ & $251(25.4)$ & $2(0.2)$ & $253(25.6)$ \\
\hline Gastrointestinal & $232(23.5)$ & $371(37.6)$ & $319(32.3)$ & $61(6.2)$ & $4(0.4)$ & $65(6.6)$ \\
\hline Urogenital & $552(55.9)$ & $334(3.8)$ & $96(9.7)$ & $5(0.5)$ & $0(0.00)$ & $5(0.50)$ \\
\hline Hematologic & $510(51.7)$ & $265(26.8)$ & $126(12.8)$ & $70(7.1)$ & $16(1.6)$ & $86(8.7)$ \\
\hline \multicolumn{7}{|l|}{ LATE TOXICITY } \\
\hline Skin & $792(80.2)$ & $183(18.5)$ & $10(1.0)$ & $2(0.2)$ & $0(0.0)$ & $2(0.2)$ \\
\hline Subcutaneous tissue & $841(85.2)$ & $130(13.2)$ & $13(1.3)$ & $2(0.2)$ & $1(0.1)$ & $3(0.3)$ \\
\hline Gastrointestinal & $699(70.8)$ & $205(20.8)$ & $65(6.6)$ & $17(1.7)$ & $1(0.1)$ & $18(1.8)$ \\
\hline Urogenital & 905 (91.7) & $65(6.6)$ & $13(1.3)$ & $3(0.3)$ & $1(0.1)$ & $4(0.4)$ \\
\hline
\end{tabular}


The most common acute toxicities were grade 2 dermatitis, reported in 491 patients $(49.75 \%)$, and grade 1 diarrhea, reported in 371 patients (37.6\%). Severe (grade $>3$ ) acute toxicity was observed mainly as skin toxicity (desquamation) in 253 patients $(25.6 \%)$. Other severe toxicities were GI in 65 patients $(6.6 \%)$ and urogenital in 5 patients $(0.5 \%)$. Overall, the grade $3-4$ acute toxicity rate was $32.7 \%$. The acute hematologic toxicity was reported in 265 patients $(26.8 \%)$ as grade 1 , in 126 patients $(12.8 \%)$ as grade 2 and in 86 patients $(8.7 \%)$ as grade $>3$ toxicity.

With a median follow-up of 28 months (range 6-138), GI late effects (diarrhea and proctitis) were the most common late toxicities: grade 1 in 205 patients $(20.8 \%)$, grade 2 in 65 patients $(6.6 \%)$ and grade $3-4$ in 18 patients (1.8\%). Grade 1, 2 and 3-4 late skin toxicities were observed in 183 patients (18.5\%), 10 patients $(1 \%)$ and 2 patients $(0.2 \%)$, respectively. Pelvic bone fractures or density alterations were reported in 16 patients $(1.6 \%)$. Sexual disorders and dyspareunia occurred in $9(0.9 \%)$ and $3(0.3 \%)$ female patients, respectively. Late anemia and/or thrombocytopenia was described in 5 patients $(0.5 \%)$.

\subsection{Treatment Response and Clinical Outcomes}

The diagnostic imaging modalities allowing for tumor response evaluation were MRI in 386 patients (39.10\%), CT in 184 patients (18.65\%), anoscopy in 146 patients $(14.80 \%)$, endorectal ultrasound (ERUS) in 98 patients (10\%) and 18FDG-PET in 71 patients (7.2\%). Multiple diagnostic imaging modalities were used in combinations in 90 patients $(9.12 \%)$.

At 3 months after the start of RT, 536 patients (54.3\%) had complete response (CR), 291 patients (29.5\%) partial response (PR), 12 patients (1.2\%) stable disease (SD), and 13 patients $(1.3 \%)$ had local progressive disease (PD). For 135 patients $(13.7 \%)$, data were not available at the 3-month analysis.

At 6 months after the start of RT, 766 patients (77.7\%) had CR, 140 patients $(14.2 \%)$ had PR, 17 patients $(1.7 \%)$ had SD, and 57 patients $(5.8 \%)$ had PD. Data were not available at the 6-month analysis for 7 patients $(0.7 \%)$. The overall clinical CR rate (beyond 6-month evaluations) was $90.6 \%$.

The 2- and 3-year LC rates were 86.9\% (95\% CI: 85.6-88.2\%) and 85.8\% (95\% CI: 84.4-87.2\%), respectively (Figure 1a).

A total of 84 patients underwent a colostomy with abdominoperineal resection in the first year after the start of RT, with an estimated cumulative incidence of colostomy at 12 months of $9.3 \%$ (95\% CI: 8.2-10.3\%). The 2- and 3-year colostomy-free survival (CFS) rates were $81.5 \%$ (95\% CI: 79.83-83.1\%) and 77.9\% (95\% CI: 76.1-79.8\%), respectively (Figure 1a).

The 2-, 3- and 5-year overall survival (OS) rates were 92.3\% (95\% CI: 91.3-93.3\%), 88.1\% (95\% CI: 78.8-89.4\%) and 82.9\% (95\% CI: 81.0-84.7\%), with 2- and 3-year progressionfree survival (PFS) rates of 83.2\% (95\% CI: 81.6-84.7\%) and 80.2\% (95\% CI: 78.5-82.0\%), respectively (Figure 1b). Overall, the 2-, 3- and 5-year event-free survival (EFS) rates were 76.6\% (95\% CI: 73.8-79.4\%), 73.4\% (95\% CI: 70.5-76.5\%) and 69.4\% (95\% CI: $66.0-73.0 \%)$, respectively. 


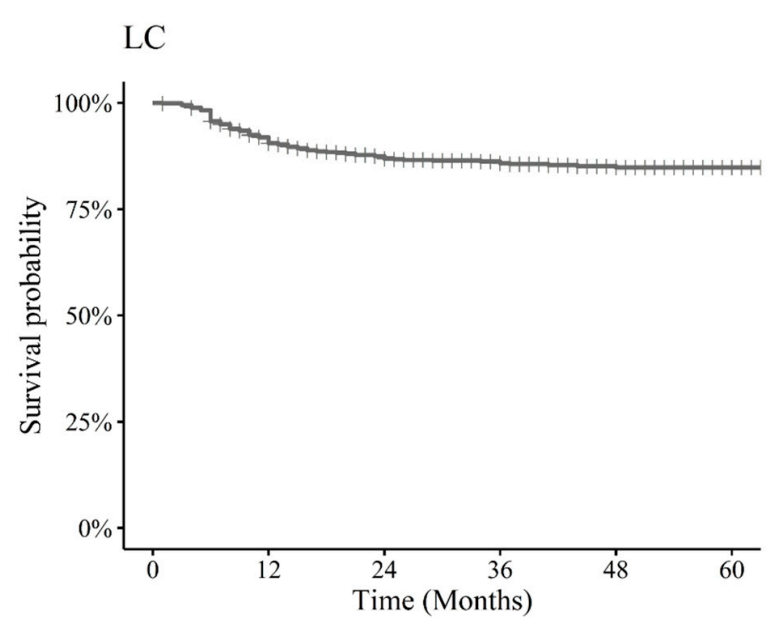

Number at risk

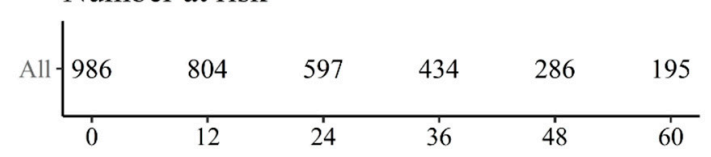

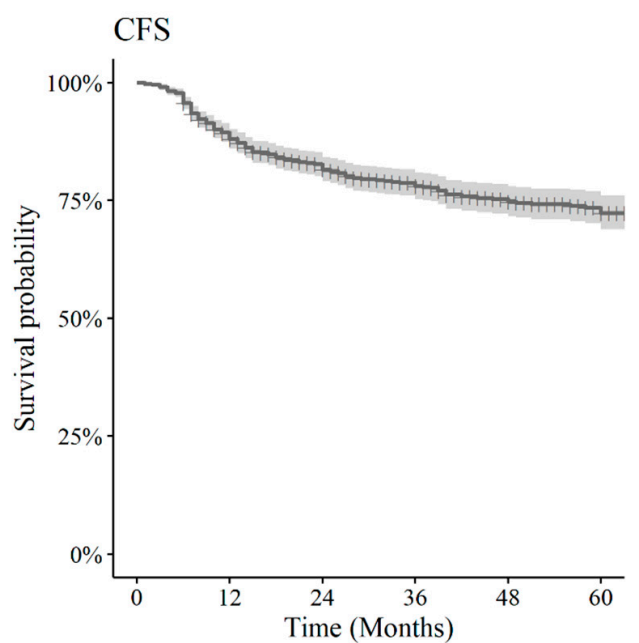

Number at risk

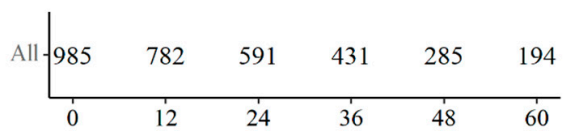

(a)
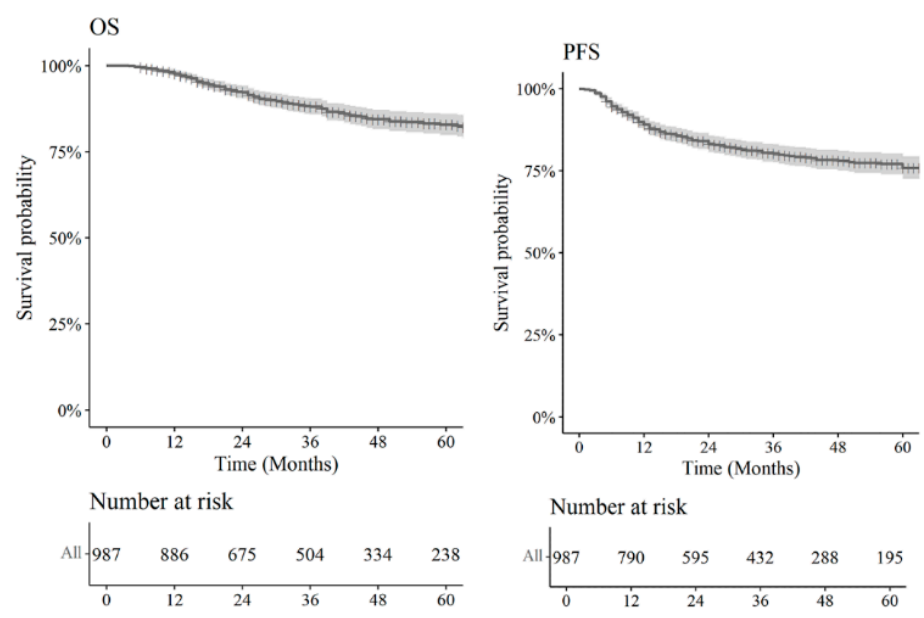

EFS
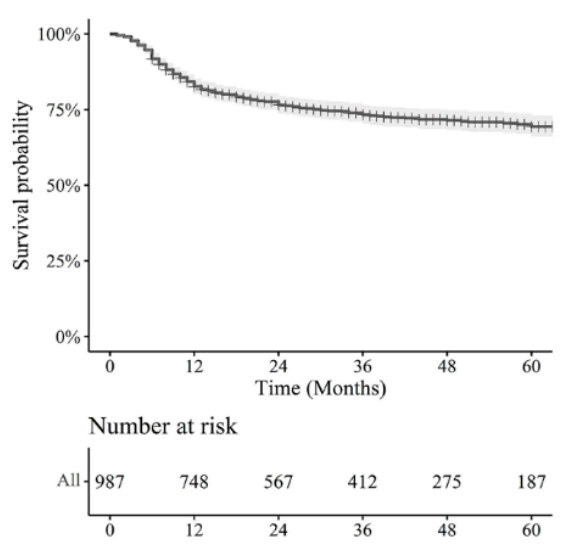

(b)

Figure 1. (a) Local control (LC), colostomy-free survival (CFS), curves and subjects at risk at 12, 24, 36, 48 and 60 months. (b) Overall survival (OS), progression-free survival (PFS), event-free survival (EFS) curves and subjects at risk at 12, 24, 36, 48 and 60 months. 


\subsection{Univariate and Multivariate Analyses}

As study variables, we evaluated age, gender, ECOG PS, HPV and HIV status, clinical stage (T1-T2 vs. T3-T4), lymph node involvement (N0 vs. N+), histological grade (G1-G2 vs. G3), and treatment parameters, such as total dose and treatment duration (OTT: $<45$ vs. $\geq 45$ days).

Univariate analysis assessing overall clinical CR (after 6 months) showed that histological grade 3 was associated with a low probability of CR (odds ratio (OR) $0.11,95 \%$ CI $0.03-0.52, p=0.030$ ). In addition, node-positive patients had a significantly lower probability of CR than patients with uninvolved lymph nodes (OR $0.45,95 \%$ CI $0.25-0.76, p=0.004$ ). Finally, a significant association with disease extension was reported (OR 0.10, 95\% CI $0.04-0.27, p<0.001$ and OR $=0.50,95 \%$ CI $0.28-0.85, p=0.013$ for extended disease (ED) and LAD, respectively).

As shown in Table 5 on univariate analysis, a statistically significant lower CFS, OS, PFS and EFS was found for patients with poor ECOG PS and an advanced stage. Lymph node involvement (regardless of T-stage) and histological grade 3 were associated with a lower LC rate $(p<0.001$ and $p=0.025$, respectively). Age $>68.5$ (cut-off set at 68.5 years as median age of population study) showed a trend for a lower PFS ( $p=0.052)$. Moreover, the correlation between clinical outcomes and disease extension is shown in Figure 2a,b.

No statistically significant association was found between RT total dose and/or boost modality (SIB) and clinical outcomes. OTT $>45$ days showed a trend with a lower PFS $(p=0.050)$ and was significantly associated with a lower EFS $(p=0.030)$ (Table 6).

LC

$$
+\mathrm{DE}-\mathrm{E}+\mathrm{DE}-\mathrm{ED}+\mathrm{DE}-\mathrm{L} A \mathrm{D}
$$

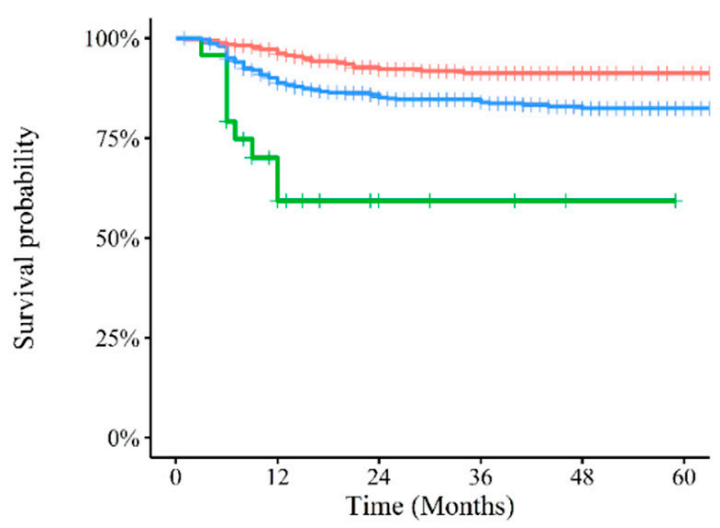

Number at risk
CFS

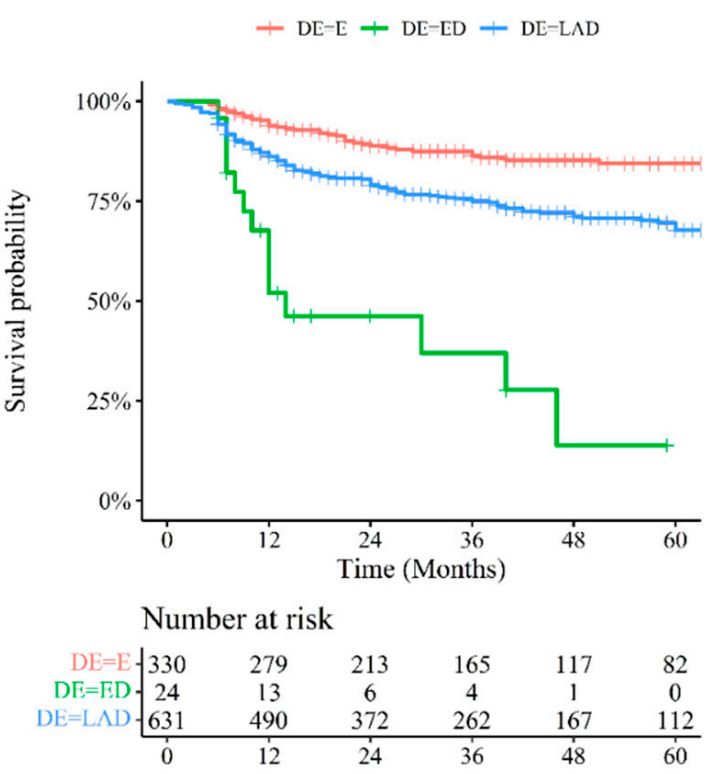

(a)

Figure 2. Cont. 
OS

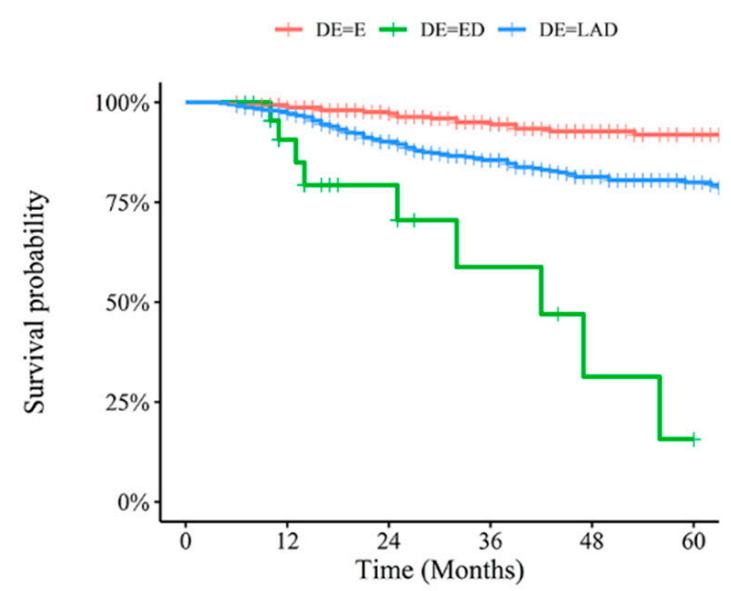

Number at risk

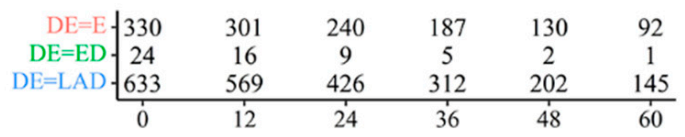

PFS

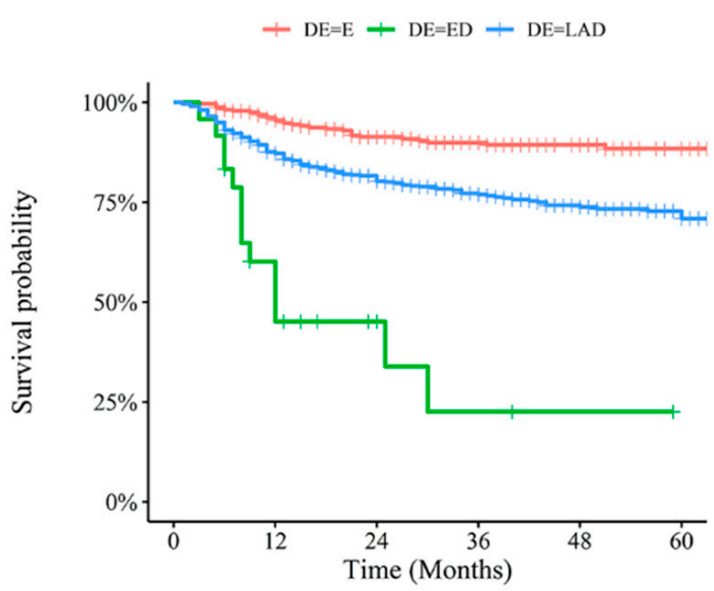

Number at risk

DE $=\mathrm{ED}=\left[\begin{array}{cccccc}330 & 284 & 217 & 164 & 114 & 78 \\ 24 & 12 & 5 & 2 & 1 & 0 \\ \mathrm{DE}=\mathrm{LAD} & \underset{633}{494} & 373 & 266 & 173 & 117 \\ \hline 0 & 12 & 24 & 36 & 48 & 60\end{array}\right.$

$\mathrm{EFS}+\mathrm{DE}=\mathrm{E}+\mathrm{DE}=\mathrm{ED}+\mathrm{DE}=\mathrm{LAD}$

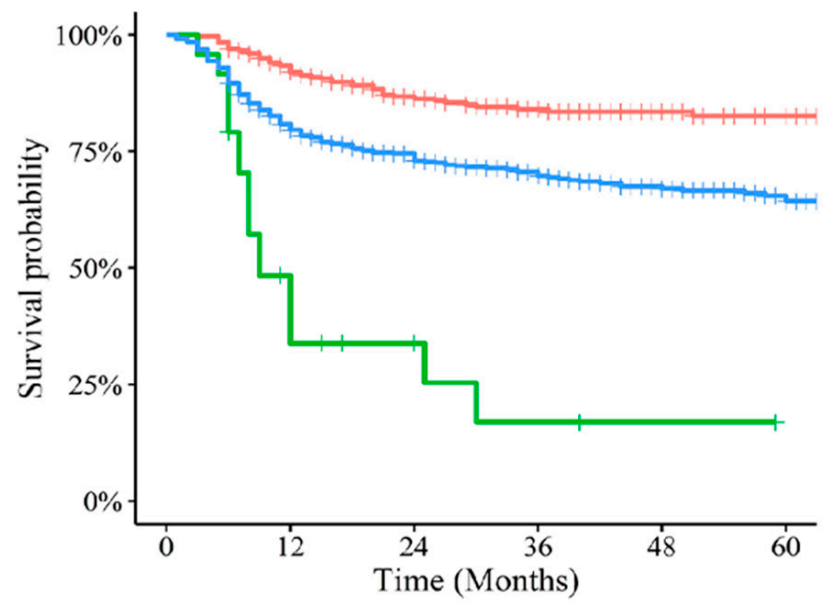

Number at risk

\begin{tabular}{|c|c|c|c|c|c|}
\hline$D E=E-330$ & 277 & 210 & 160 & 113 & 78 \\
\hline $\mathrm{DE}=\mathrm{ED}-24$ & 10 & 5 & 2 & 1 & 0 \\
\hline$D E=L A D-633$ & 461 & 352 & 250 & 161 & 109 \\
\hline 0 & 12 & 24 & 36 & 48 & 60 \\
\hline
\end{tabular}

(b)

Figure 2. (a) Kaplan-Meier survival curves stratified by disease extension and risk table at 12, 24, 36, 48 and 60 months for local control (LC) and colostomy-free survival (CFS). Early disease: T1-T2 tumors; LAD = locally advanced disease (T3-T4 or $\mathrm{N}+=$ node-positive tumors); ED = extended disease (lumbo-aortic and/or common iliac lymph nodes). (b) Kaplan-Meier survival curves stratified by disease extension and risk table at 12, 24, 36, 48 and 60 months for overall survival (OS), progression-free survival (PFS) and event-free survival (EFS). Early disease: T1-T2 tumors; LAD = locally advanced disease (T3-T4 or $\mathrm{N}+=$ node-positive tumors); ED = extended disease (lumbo-aortic and/or common iliac lymph nodes). 
Table 5. Univariate analysis between patient characteristics and clinical outcomes.

\begin{tabular}{|c|c|c|c|c|c|c|c|c|c|c|c|c|c|c|c|}
\hline \multirow{2}{*}{ Variable } & \multicolumn{3}{|c|}{ LC } & \multicolumn{3}{|c|}{ CFS } & \multicolumn{3}{|c|}{ OS } & \multicolumn{3}{|c|}{ PFS } & \multicolumn{3}{|c|}{ EFS } \\
\hline & HR & $95 \% \mathrm{CI}$ & $p$-Value & HR & $95 \% \mathrm{CI}$ & $p$-Value & HR & $95 \% \mathrm{CI}$ & $p$-Value & HR & $95 \% \mathrm{CI}$ & $p$-Value & HR & $95 \% \mathrm{CI}$ & $p$-Value \\
\hline $\begin{array}{l}\text { Gender (ref. Male) } \\
\text { Female }\end{array}$ & 0.77 & $(0.54-1.09)$ & 0.160 & 0.73 & $(0.54-0.96)$ & 0.026 & 0.65 & $(0.45-0.94)$ & 0.022 & 0.74 & $(0.54-0.98)$ & 0.050 & 0.75 & $(0.58-0.97)$ & 0.031 \\
\hline $\begin{array}{l}\text { Age (ref. }<68.5 \text { year) } \\
\geq 68.5 \text { year }\end{array}$ & 0.88 & $(0.62-1.25)$ & 0.491 & 1.07 & $(0.82-1.40)$ & 0.604 & 1.33 & $(0.93-1.89)$ & 0.111 & 1.33 & $(1.00-1.78)$ & 0.050 & 1.15 & $(0.90-1.47)$ & 0.276 \\
\hline $\begin{array}{c}\text { ECOG PS (ref. 0) } \\
1 \\
2\end{array}$ & $\begin{array}{l}1.45 \\
1.60\end{array}$ & $\begin{array}{l}(0.96-2.16) \\
(0.392 .16)\end{array}$ & $\begin{array}{l}0.071 \\
0.511\end{array}$ & $\begin{array}{l}1.43 \\
2.50\end{array}$ & $\begin{array}{l}(1.04-1.96) \\
(1.02-6.09)\end{array}$ & $\begin{array}{l}0.025 \\
0.044\end{array}$ & $\begin{array}{l}1.59 \\
4.23\end{array}$ & $\begin{array}{l}(1.06-2.38) \\
(1.55-11.54)\end{array}$ & $\begin{array}{l}0.025 \\
0.005\end{array}$ & $\begin{array}{l}1.55 \\
2.31\end{array}$ & $\begin{array}{l}(1.12-2.16) \\
(0.85-6.26)\end{array}$ & $\begin{array}{l}\mathbf{0 . 0 0 9} \\
0.098\end{array}$ & $\begin{array}{l}1.47 \\
2.02\end{array}$ & $\begin{array}{l}(1.10-1.95) \\
(0.83-4.91)\end{array}$ & $\begin{array}{l}\mathbf{0 . 0 0 8} \\
0.121\end{array}$ \\
\hline $\begin{array}{l}\text { HIV (ref. no) } \\
\text { Yes }\end{array}$ & 1.29 & $(0.73-2.26)$ & 0.381 & 1.39 & $(0.92-2.08)$ & 0.117 & 2.06 & $(1.28-3.28)$ & 0.003 & 1.67 & $(1.10-2.52)$ & 0.015 & 1.35 & $(0.93-1.98)$ & 0.118 \\
\hline $\begin{array}{c}\text { HPV (ref. No) } \\
\text { Yes }\end{array}$ & 1.11 & $(0.66-1.87)$ & 0.689 & 1.01 & $(0.65-1.54)$ & 0.976 & 1.34 & $(0.74-2.42)$ & 0.327 & 0.91 & $(0.57-1.43)$ & 0.671 & 0.90 & $(0.61-1.32)$ & 0.595 \\
\hline $\begin{array}{c}\text { Histology (ref. Squamous) } \\
\text { Basaloid }\end{array}$ & 0.66 & $(0.32-1.35)$ & 0.255 & 0.73 & $(0.43-1.23)$ & 0.242 & 0.74 & $(0.37-1.45)$ & 0.379 & 0.75 & $(0.43-1.32)$ & 0.320 & 0.76 & $(0.47-1.22)$ & 0.256 \\
\hline $\begin{array}{c}\text { Histological Grade (ref. 1) } \\
2 \\
3\end{array}$ & $\begin{array}{l}2.60 \\
3.83\end{array}$ & $\begin{array}{l}(0.80-8.42) \\
(1.18-12.42)\end{array}$ & $\begin{array}{l}0.111 \\
\mathbf{0 . 0 2 5}\end{array}$ & $\begin{array}{l}0.97 \\
0.97\end{array}$ & $\begin{array}{l}(0.55-1.68) \\
(0.54-1.72)\end{array}$ & $\begin{array}{l}0.904 \\
0.917\end{array}$ & $\begin{array}{l}1.03 \\
1.14\end{array}$ & $\begin{array}{l}(0.50-2.10) \\
(0.55-2.39)\end{array}$ & $\begin{array}{l}0.941 \\
0.720\end{array}$ & $\begin{array}{l}1.07 \\
1.32\end{array}$ & $\begin{array}{l}(0.57-1.98) \\
(0.70-2.47)\end{array}$ & $\begin{array}{l}0.837 \\
0.391\end{array}$ & $\begin{array}{l}1.07 \\
1.17\end{array}$ & $\begin{array}{l}(0.63-1.83) \\
(0.67-2.02)\end{array}$ & $\begin{array}{l}0.800 \\
0.582\end{array}$ \\
\hline $\begin{array}{c}\text { Disease extension (ref. Early) } \\
\text { ED } \\
\text { LAD }\end{array}$ & $\begin{array}{l}7.59 \\
2.12\end{array}$ & $\begin{array}{c}(3.53-16.31) \\
(1.36-3.29)\end{array}$ & $\begin{array}{l}<0.001 \\
0.001\end{array}$ & $\begin{array}{l}7.90 \\
2.14\end{array}$ & $\begin{array}{c}(4.30-14.50) \\
(1.52-2.99)\end{array}$ & $\begin{array}{l}<0.001 \\
<0.001\end{array}$ & $\begin{array}{c}12.50 \\
2.78\end{array}$ & $\begin{array}{c}(5.66-27.60) \\
(1.71-4.50)\end{array}$ & $\begin{array}{l}<0.001 \\
<0.001\end{array}$ & $\begin{array}{c}11.27 \\
2.55\end{array}$ & $\begin{array}{c}(5.98-21.22) \\
(1.74-3.74)\end{array}$ & $\begin{array}{l}<0.001 \\
<0.001\end{array}$ & $\begin{array}{l}8.44 \\
2.22\end{array}$ & $\begin{array}{c}(4.85-14.69) \\
(1.62-3.03)\end{array}$ & $\begin{array}{l}<0.001 \\
<0.001\end{array}$ \\
\hline
\end{tabular}

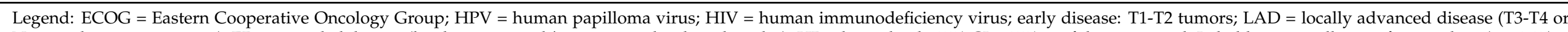

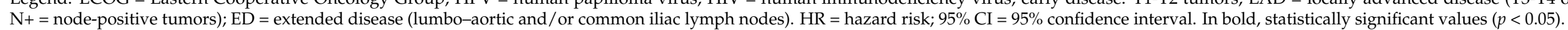


Table 6. Univariate analysis treatment characteristics and clinical outcomes.

\begin{tabular}{|c|c|c|c|c|c|c|c|c|c|c|c|c|c|c|c|}
\hline Variable & & LC & & & CFS & & & OS & & & PFS & & & EFS & \\
\hline & HR & $95 \% \mathrm{CI}$ & $p$-Value & HR & $95 \%$ CI & $p$-Value & HR & $95 \% \mathrm{CI}$ & $p$-Value & HR & $95 \% \mathrm{CI}$ & $p$-Value & HR & $95 \%$ CI & $p$-Value \\
\hline $\begin{array}{l}\text { OTT (Ref. <45) } \\
\quad \geq 45\end{array}$ & 1.13 & $(0.80-1.61)$ & 0.478 & 1.22 & $(0.93-1.60)$ & 0.140 & 1.23 & $(0.86-1.75)$ & 0.243 & 1.33 & (1.00-1.77) & 0.050 & 1.31 & $(1.03-1.68)$ & 0.030 \\
\hline $\begin{array}{c}\text { Total dose } 54 \text { Gy (ref. } \leq 54 \text { Gy) } \\
>54 \text { Gy }\end{array}$ & 0.81 & $(0.57-1.15)$ & 0.238 & 0.90 & $(0.69-1.18)$ & 0.463 & 0.85 & $(0.60-1.22)$ & 0.383 & 1.03 & $(0.77-1.39)$ & 0.821 & 0.98 & $(0.76-1.26)$ & 0.882 \\
\hline $\begin{array}{c}\text { Total dose } 55 \text { Gy (ref. } \leq 55 \text { Gy) } \\
>55 \text { Gy }\end{array}$ & 0.81 & $(0.57-1.15)$ & 0.238 & 0.90 & $(0.69-1.18)$ & 0.463 & 0.85 & $(0.60-1.22)$ & 0.383 & 1.03 & $(0.77-1.39)$ & 0.821 & 1.02 & $(0.79-1.30)$ & 0.904 \\
\hline $\begin{array}{c}\text { Dose/Fraction HR PTV (ref. } \\
1.8-2 \mathrm{~Gy}) \\
>2 \mathrm{~Gy})\end{array}$ & 0.79 & $(0.56-1.12)$ & 0.187 & 0.89 & $(0.67-1.17)$ & 0.385 & 0.88 & $(0.61-1.25)$ & 0.471 & 0,93 & $(0.69-1.25)$ & 0.647 & 0.91 & $(0.71-1.17)$ & 0.452 \\
\hline $\begin{array}{c}\text { Dose/Fraction LR PTV (ref. } \\
1.8-2 \mathrm{~Gy}) \\
<1.8 \mathrm{~Gy}\end{array}$ & 0.96 & $(0.67-1.39)$ & 0.835 & 0.98 & $(0.74-1.31)$ & 0.908 & 0.74 & $(0.52-1.06)$ & 0.102 & 0.78 & $(0.58-1.04)$ & 0.090 & 1.01 & $(0.67-1.51)$ & 0.972 \\
\hline $\begin{array}{l}\text { SIB (ref. No) } \\
\text { Yes }\end{array}$ & 0.92 & $(0.64-1.30)$ & 0.639 & 0.92 & $(0.70-1.20)$ & 0.527 & 0.94 & (0.65-1.33) & 0.713 & 0.93 & $(0.69-1.24)$ & 0.616 & 0.89 & $(0.69-1.13)$ & 0.334 \\
\hline
\end{tabular}

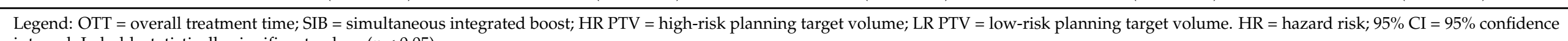
interval. In bold, statistically significant values $(p<0.05)$. 
According to the multivariate analysis, lymph node involvement negatively affected all clinical outcome measures (LC, CFS, OS, PFS and EFS). Age $>68.5$ (cut-off set at 68.5 years as median age of population study) and pathological grade 3 were confirmed as negative prognostic factors for PFS $(p=0.019)$ and LC $(p=0.032)$, respectively.

Investigating the relationships between toxicity (all grades) and the clinical and dosimetric parameters due to the high dimensionality of the comparisons, we calculated ORs and CIs (95\%) considering a significant $p$-value of $p<0.001$. In particular, disease extension results were associated with acute skin toxicity (ED vs. Early: OR $=4.84,95 \%$ CI 2.13-12.04; LAD vs. Early: OR $=0.60,95 \%$ CI $0.46-0.76$ ), acute gastrointestinal toxicity (LAD vs. Early: $\mathrm{OR}=0.51,95 \% \mathrm{CI} 0.4-0.65$ ) and with acute urological toxicity (LAD vs. Early: OR $=0.36$, 95\% CI 0.27-0.46). Disease extension results were also associated with skin (ED vs Early: $\mathrm{OR}=19.64,95 \%$ CI 8.3-48.63) and subcutaneous late toxicity (LAD vs. Early: OR = 0.43, 95\% CI 0.3-0.61).

\section{Discussion}

CRT provided high rates of complete responders (up to 80-90\%) in several trials [1-9] and currently represents the standard treatment for stage II-III AC patients. Radiotherapy delivery in anal cancer is complex because of the target shape and the proximity to dose-sensitive organs at risk (OARs), such as bowel, femoral heads, bladder, genitalia and perineal skin. IMRT techniques, modulating the beam fluence during delivery, offer the possibility of a dose-painted treatment with high doses to the tumor, minimizing the dose to surrounding OARs. This potentially allows the reduction of acute and late adverse events, improving the therapeutic ratio. The consequent decrease in acute toxicity improves treatment compliance, with a decrease in interruptions and a shorter OTT, with a potential beneficial effect on clinical outcomes. Several studies have shown promising results using this approach [12-22], and it should be considered the standard of care for anal cancer [23].

In our study, all patients were treated with IMRT techniques.

We observed a substantial clinical response rate: $6-\mathrm{moCR}$ of $77.7 \%$ and an overall clinical CR rate (beyond 6-month evaluations) of $90.6 \%$, in agreement with previous studies.

The ACT II trial reported a 26-week CR rate of $89.6 \%$ in the cisplatin group and $90.5 \%$ in the MMC group, showing that patients with $\mathrm{CCR}$ at 26 weeks had a superior 5-year OS compared to patients without CCR [7]. Based on these data, the European Society for Medical Oncology (ESMO) guidelines recommend a clinical evaluation for CR and endorsed a watchful wait approach, stating that partial regression can be managed by a close follow-up to confirm that complete regression occurs within 6 months [23].

Five-year OS in patients with stage II-III anal squamous cell cancer, treated with CRT, is approximately $75 \%$. RTOG 9811 and ACT II used conformal RT techniques with concurrent 5-FU and MMC and reported 5-year OS rates of 78.3\% and 79.0\%, respectively [6,8,9]. Long term results (median follow-up of 49 months) of a single institution study of 99 patients, treated with dose-painted IMRT according to the RTOG 0529 trial, showed a 4 -year OS of $85.8 \%$ [17]. Moreover, in an Italian mono-institutional cohort of 87 patients treated with SIB-IMRT, the 3-year OS rate was 79\%, with a 3-year CFS and LC rate of $64 \%$ and $69 \%$, respectively [18]. In our study, the estimated 3- and 5-year OS rates were $88.09 \%$ (95\% CI: 78.76-89.42) and 82.86\% (95\% CI: 81-84.72), respectively. Consistent results in terms of 3-year LC (85.84\%; 95\% CI: 84.43-87.25), 3-year CFS (77.94\%; 95\% CI: 76.07-79.81) and 3-year PFS (80.24; 95\% CI: 78.5-81.98) rates were also observed.

Several trials reported that high conformal techniques, such as IMRT, VMAT and tomotherapy, require an accurate definition and delineation of treatment volumes to maintain or potentially improve locoregional control. In AC patients, the primary tumor site, pelvic and inguinal nodes are the main areas of recurrence and could represent three different targets for RT planning. Recommendations based on expert views concerning the primary tumor and elective lymph nodes delineation have been provided [24,25]. On the other hand, no consensus is available from randomized trials regarding the optimal total dose and daily fractionation in the different disease stages, although early-stage disease 
often receives lower doses, while larger and more advanced tumors are treated with higher doses [1-8].

Data from randomized trials showed that CRT with relatively low total radiation doses (30-50 Gy) and the addition of MMC to 5FU resulted in high LC rates in small tumors $(<4 \mathrm{~cm})[1,2]$. An LC rate ranging between $45-55 \%$ has been reported with a modest dose of $45 \mathrm{~Gy}$ in 25 fractions and the boost dose modulated according to treatment response [1-4]. In the RTOG 8704, 9811 phase III trials (median RT dose of 48 Gy, without planned gap) in patients with T3, T4, node-positive disease and residual tumor, an additional boost of 9 to $14 \mathrm{~Gy}$ (2.0 Gy/daily, total dose of 54 to $59 \mathrm{~Gy}$ ) was delivered to the primary tumor/nodal mass [4,6]. Prophylactic irradiation of inguinal lymph nodes up to a dose of $45 \mathrm{~Gy}$ increased $\mathrm{LC}$ and is recommended, but it remains unclear whether boosting the radiation dose to $>50$ Gy in patients with good response will improve the results $[4,6]$. Higher doses above 56 Gy may provide better local control, but they could be associated with an increased toxicity [26], and doses $>59$ Gy were not shown to provide additional benefits [27].

In our study, patients were treated with a median total dose of 55 Gy (range: 45-75). SIB was delivered in 568 patients ( $57.5 \%$ ), and an additional sequential EBRT or BRT boost was administered in 167 patients (16.9\%). Concomitant chemotherapy was administered in all patients with MMC plus 5-FU or the capecitabine regimen in 779 patients $(78.92 \%)$.

The treatment was well tolerated, reporting grade 3 acute skin, GI and urogenital toxicity in 251 patients $(25.4 \%), 61$ patients $(6.2 \%)$ and 5 patients $(0.5 \%)$, respectively. In RTOG 0529, aiming to evaluate the tolerability of a dose-painted IMRT treatment, acute $\geq \mathrm{G} 3$ skin and gastrointestinal toxicity rates were $23 \%$ and $21 \%$, respectively [15] Overall, our analysis showed grade $3-4$ acute toxicity in $32.7 \%$ of patients, related mainly to local skin toxicity $(25.6 \%)$. Grade $3-4$ acute hematologic toxicity in our study was also limited $(8.7 \%)$, despite the fact that selective approaches to spare the bone marrow were not routinely used in some institutions [28].

The good toxicity profile seems noteworthy considering the median OTT of 45 days (range: 25-115), with a treatment interruption of $>5$ days for toxicity in 186 patients (18.8\%). Nine hundred twenty patients (93.2\%) completed the planned RT treatment with a high compliance to treatment. These data confirmed the treatment tolerability with a low rate of interruptions with IMRT techniques, even when SIB is delivered with consequent reduced OTT.

The SIB strategy, allowing for both gross tumor volume and elective volumes to receive different total doses in the same number of fractions, could require that different elective nodal volumes are treated with a varying dose per fraction $[14,15]$. Historically, anal cancer has been treated with doses of 1.8 Gy per fraction, using a shrinking field technique over the course of treatment. Using IMRT with SIB to treat different target volumes with different daily doses often results in some areas receiving less than conventional fractional doses of radiation (i.e., $<1.80 \mathrm{~Gy}$ ). In the phase II RTOG 0529 study [15], some patients received, to some elective nodal volumes, a fractional dose of $1.5 \mathrm{~Gy}$ without loss of effectiveness in LC and disease-free survival rates compared to RTOG 9811 [6]. The median OTT in the study was 43 days compared with 49 days in the RTOG 9811.

In AC patients, the reduction of OTT could lead to a clinical benefit, whereas a longer OTT could be detrimental to the likelihood of receiving a colostomy and LC $[10,11]$ due to an accelerated repopulation that could occur after irradiation and may lead to a loss in tumor control [29]. Data pooling from the RTOG-8704 and the RTOG-9811 studies showed that OTT was positively associated with time to colostomy failure but not with OS or CFS [10]. Although our analysis did not show a significant correlation between OTT prolongation and CR rate, a detrimental trend effect was observed between OTT $>45$ days and PFS $(p=0.050)$ and was significantly associated with a lower EFS $(p=0.030)$, confirming the benefit of "gap" avoidance techniques, such as IMRT-SIB [11].

Recently, according to the UK IMRT guidance [30], a consecutive cohort of 385 patients was retrospectively analyzed. All treatments were delivered by SIB in 28 fractions, with T1$2 \mathrm{~N} 0$ receiving $50.4 \mathrm{~Gy}$ to the gross primary tumor and T1-2 N+ or T3-4 N-any receiving 
53.2 Gy. Involved nodes received 50.4 Gy if $3 \mathrm{~cm}$ large; uninvolved pelvic nodes (PTVelective), including mesorectal, obturator, external and internal iliac, inguinal and presacral, received 40 Gy since March 2014 (dose per fraction: 1.4 Gy). As reported by the authors, this was a biologically equivalent dose to $30.6 \mathrm{~Gy}$ in 17 fractions delivered in the ACT2 trial, using an alpha-beta ratio of 8 and a loss of 0.7 Gy per day after 20 fractions. Only two isolated lymph node relapses have been reported with this low dose per fraction approach on elective volume [31]; then, the efficacy of doses less than 1.80 Gy per day are assumed.

Parallel to these studies, in our cohort the elective low-risk PTV received less than 1.80 Gy per day in more than half of the patients. No statistically significant correlation was found between boost modality (SIB), total RT dose (grouping patients receiving less than, equal to and more than 54 and 55 Gy) or dose per fraction (grouping patients receiving hazard ratio (HR) PTV less than, equal to and more than 2 Gy/fraction, and LR PTV less than, equal to and more than 1.8-2 Gy/fraction) and clinical response and outcomes.

Even though a certain heterogeneity in the dose volume prescription of elective PTVs has been reported in our study, this factor did not affect treatment response, and the several RT schedules used seem to be equivalent in terms of clinical outcomes. Based on these results, although recommendations about optimal total dose and fractionation cannot be provided, our study confirmed that low fractional doses may be appropriate for clinically negative areas when using IMRT for AC with concurrent chemotherapy. The ongoing PLATO integrated protocol looking at dose escalation in LAD and dose de-escalation in early small-node-negative disease is currently set up in the UK, and in the future will inform dose fraction optimization for AC. Finally, we are currently performing a national survey to explore the gray areas in the pattern of care of $\mathrm{AC}$ and to define a clinical practice consensus, especially in terms of treatment doses and volumes.

Data on the HPV status of more than half of our patients were missing, which is probably related to the retrospective nature of the study, and this could explain the positive/negative HPV-rate in our analysis. Indeed, AC is reported to be associated with HPV infection in 70-90\% of cases, with HPV16 as the most common sub-type. This could also have had an impact on the non-statistically significant correlation with clinical outcomes in the two subgroups.

Histological grade 3 and lymph node involvement (with any T) were associated with a lower probability of CR (OR $0.11,95 \%$ CI $0.03-0.52, p=0.030$ and OR $0.45,95 \%$ CI $0.25-0.76, p=0.004$, respectively) and lower LC ( $p<0.001$ and $p=0.025$, respectively). Lymph node involvement remained negatively associated with all clinical outcomes (LC, CFS, OS, PFS and EFS) also in the multivariate analysis. Advanced stage was confirmed to be a negative prognostic factor for CFS, OS, PFS and EFS. Furthermore, as reported in other studies, nodal involvement and advanced stage results were significant predictors for LC, OS and PFS [32]. Moreover, disease extension results, probably due to large volumes treated at high doses, were associated with higher risk of toxicity.

\section{Materials and Methods}

The study was designed as a retrospective research project named "RAINSTORM: RAdiotherapy with INtenSiTy mOdulated (IMRT) techniques in the treatment of anal caRcinoMa: a multicenter retrospective observation study" approved by the Ethical Committee of the "SS Annunziata" Hospital, "G. D'Annunzio" University, Chieti, Italy, and all joined centers.

All patients had histologically confirmed AC (both anal canal and margin). Pretreatment data, including age, gender, performance status as per Eastern Cooperative Oncology Group (ECOG), histological type, human papilloma virus (HPV) and human immunodeficiency virus (HIV) status, were collected. For diagnosis and staging, computed tomography scan (CT), endorectal ultrasound (ERUS), magnetic resonance imaging (MRI) and 18F-fluorodeoxyglucose positron emission tomography (18FDG-PET) were employed. Patients were staged according to the UICC TNM staging system 6th edition 2002 and 7th edition 2009 and classified in early disease (E: T1-2N0) and locally advanced disease (LAD: 
T3-4 or N+). Patients with T1N0 tumors of the anal margin were excluded in case they received local excision. Patients with a single site of clinical abdominal lymph node involvement (lumbar-aortic and / or common iliac lymph nodes) were included and classified in extended disease (ED).

\subsection{Treatment Characteristics}

The total radiotherapy dose and daily fractionation prescription were investigated according to the planning target volumes (PTVs) as defined in the RTOG 0529 study [15] (low-, intermediate- and high-risk volumes) and analyzed based on clinical stage at presentation. All patients were treated with one of the IMRT modalities available in their center: static or dynamic IMRT, volumetric modulated arc therapy (VMAT) or helical tomotherapy (HT). Timing and modalities of further boost were also investigated. Detailed data concerning concomitant chemotherapy were collected.

\subsection{Statistical Analysis}

The primary endpoints were the clinical complete response rate at 6 months (6-moCR) and colostomy-free survival (CFS). CFS was defined as the time between the start of CRT and the date of colostomy, death or last follow-up in which the patient was known to be colostomy-free. Secondary endpoints were overall survival (OS) and acute and late toxicity. OS was calculated in all patients from the date of diagnosis to the date of death from any cause or the last follow-up; toxicities were retrospectively graded according to Common Terminology Criteria for Adverse Events (CTCAE) Version 4.0 by the treating physicians [33]. Acute toxicities were defined as occurring within 90 days of treatment and late toxicities as occurring after 90 days.

Tumor response assessment was investigated at 3 and 6 months after the start of CRT and evaluated with the response evaluation criteria in solid tumors (RECIST) v1.1 [34]. LC was defined as the time between the start of CRT and the date of the first documented tumor recurrence or persistent local disease after CRT. Progression-free survival (PFS) was defined as all progressive diseases, local recurrence, metastases, or death from any cause $[35,36]$. Event-free survival (EFS) was then calculated for all documented events (local recurrence, metastases, colostomy or death). For patients in which no event occurred, we defined the follow-up time interval as the time elapsed until the last scheduled followup visit.

The univariate ordinal logistic regressions and binomial logistic regressions were used to study the relationships between toxicities and the main variables of interest. The results of models were expressed as an odds ratio (OR) and a relative $95 \%$ confidence interval $(95 \%$ $\mathrm{CI}$ ). Due to the high dimensionality of the comparisons, we considered a $p$-value $<0.001$.

Univariate logistic regression models were applied to determine the study variables predictive of the clinical complete response rate at 6 months (6-moCR). The results of models were expressed as an odds ratio (OR) and a relative $95 \%$ confidence interval $(95 \%$ CI). The Kaplan-Meier method was used to calculate the rates of LC, CFS, OS, PFS and EFS at different time points. Univariate and multivariate analyses were performed using the Cox proportional hazards model to determine independent prognostic factors that had a significant impact on clinical outcomes. Calculating the exponential of the regression coefficients from the Cox model provided an estimate of the hazard ratio (HR) and the $95 \%$ confidence interval $(95 \% \mathrm{CI})$. Multicollinearity between variables was also tested using the variation inflation factor (VIF). The stability of the models was guaranteed by backward fitting procedure. All statistical analyses were performed using R statistical software (version 3.1.2.; R Foundation for Statistical Computing, Vienna, Austria). All $p$-values were two-tailed and a $p$-value $<0.05$ was considered indicative of a statistically significant association. 


\section{Conclusions}

Related to its retrospective design, our study has some limitations, such as RT dose heterogeneity and boost delivery modality reporting. Moreover, given the variable followup, there may have been difficulty in analyzing toxicity rates, particularly in reporting acute hematological toxicity, compliance to chemotherapy and overall late toxicities.

On the other hand, to our knowledge, this analysis reports the largest cohort of patients treated with IMRT techniques showing an excellent clinical result and a good toxicity profile, confirming IMRT techniques as standard of care for curative treatment of anal cancer patients. Disease extension results associated with higher risk of toxicity and lymph node involvement and high histological grade have been confirmed as the most impactful negative prognostic factors for complete clinical response in LC, CFS, OS, PFS and EFS.

Author Contributions: Conceptualization, L.C., G.M., F.V. and D.G.; methodology, L.C., G.M., F.V., A.P. and M.D.N.; software, L.C., C.R., L.G. and A.P.; validation, G.M., F.V., D.G. and M.D.N.; formal analysis, L.C., A.P. and M.D.N.; investigation, all authors; data curation, all authors; writing-original draft preparation, L.C., C.R., L.G., A.P. and M.D.N.; writing-review and editing, all authors; visualization and approval, all authors. All authors have read and agreed to the published version of the manuscript.

Funding: This research received no external funding. One authors' institution (MAG, IEO European Institute of Oncol) receives Ricerca Corrente and $5 \times 1000$ research funds from the Italian Ministry of Health. The sponsor did not play any role in study design, execution and data analysis.

Institutional Review Board Statement: The study was conducted according to the guidelines of the Declaration of Helsinki, and approved by the Ethics Committee of the "SS Annunziata" Hospital, “G. D'Annunzio" University, Chieti, Italy, with ethic approval code n¹856, on 3 December 2018.

Informed Consent Statement: Not applicable.

Data Availability Statement: Not applicable.

Acknowledgments: The Authors thank the Scientific Committee and Board of the AIRO for the critical revision and final approval of the paper.

Conflicts of Interest: The authors declare no conflict of interest.

\section{References}

1. Nigro, N.D.; Seydel, H.G.; Considine, B.; Vaitkevicius, V.K.; Leichman, L.; Kinzie, J.J. Combined preoperative radiation and chemo-therapy for squamous cell carcinoma of the anal canal. Cancer 1983, 51, 1826-1829. [CrossRef]

2. Cummings, B.; Keane, T.; O'Sullivan, B.; Wong, C.; Catton, C. Epidermoid anal cancer: Treatment by radiation alone or by radiation and 5-fluorouracil with and without mitomycin C. Int. J. Radiat. Oncol. 1991, 21, 1115-1125. [CrossRef]

3. Arnott, S.J.; Cunningham, D.; Gallagher, J.; Gray, R.; Hardcastle, J.; Houghton, J.; James, R.D.; Lennon, T.A.; Meadows, H.M.; Mossman, J.; et al. Epidermoid anal cancer: Results from the UKCCCR randomised trial of radiotherapy alone versus radiotherapy, 5-fluorouracil, and mitomycin. UKCCCR Anal Cancer Trial Working Party. UK Co-ordinating Committee on Cancer Research. Lancet 1996, 348, 1049-1054.

4. $\quad$ Flam, M.; John, M.; Pajak, T.F.; Petrelli, N.; Myerson, R.; Doggett, S.; Quivey, J.; Rotman, M.; Kerman, H.; Coia, L.; et al. Role of mitomycin in combination with fluorouracil and radiotherapy, and of salvage chemoradiation in the definitive nonsurgical treatment of epidermoid carcinoma of the anal canal: Results of a phase III randomized intergroup study. J. Clin. Oncol. 1996, 14, 2527-2539. [CrossRef] [PubMed]

5. Bartelink, H.; Roelofsen, F.; Eschwege, F.; Rougier, P.; Bosset, J.F.; Gonzalez, D.G.; Peiffert, D.; Van Glabbeke, M.; Pierart, M. Concomitant radiotherapy and chemotherapy is superior to radiotherapy alone in the treatment of locally advanced anal cancer: Results of a phase III randomized trial of the European Organization for Research and Treatment of Cancer Radiotherapy and Gastrointestinal Cooperative Groups. J. Clin. Oncol. 1997, 15, 2040-2049. [CrossRef]

6. Ajani, J.A.; Winter, K.A.; Gunderson, L.L.; Pedersen, J.; Benson, A.B.; Thomas, C.R.; Mayer, R.J.; Haddock, M.G.; Rich, T.A.; Willett, C. Fluorouracil, Mitomycin, and Radiotherapy vs Fluorouracil, Cisplatin, and Radiotherapy for Carcinoma of the Anal Canal: A randomized controlled trial. JAMA 2008, 299, 1914-1921. [CrossRef]

7. Peiffert, D.; Tournier-Rangeard, L.; Gérard, J.-P.; Lemanski, C.; François, E.; Giovannini, M.; Cvitkovic, F.; Mirabel, X.; Bouché, O.; Luporsi, E.; et al. Induction Chemotherapy and Dose Intensification of the Radiation Boost in Locally Advanced Anal Canal Carcinoma: Final Analysis of the Randomized UNICANCER ACCORD 03 Trial. J. Clin. Oncol. 2012, 30, 1941-1948. [CrossRef] 
8. James, R.D.; Glynne-Jones, R.; Meadows, H.M.; Cunningham, D.; Myint, A.S.; Saunders, M.P.; Maughan, T.; McDonald, A.; Essapen, S.; Leslie, M.; et al. Mitomycin or cisplatin chemoradiation with or without maintenance chemotherapy for treatment of squamous-cell carcinoma of the anus (ACT II): A randomised, phase 3, open-label, $2 \times 2$ factorial trial. Lancet Oncol. 2013, 14, 516-524. [CrossRef]

9. Gunderson, L.L.; Winter, K.A.; Ajani, J.A.; Pedersen, J.E.; Moughan, J.; Benson, A.B., 3rd; Thomas, C.R., Jr.; Mayer, R.J.; Haddock, M.G.; Rich, T.A.; et al. Long-Term Update of US GI Intergroup RTOG 98-11 Phase III Trial for Anal Carcinoma: Survival, Relapse, and Colostomy Failure with Concurrent Chemoradiation Involving Fluorouracil/Mitomycin Versus Fluorouracil/Cisplatin. J. Clin. Oncol. 2012, 30, 4344-4351. [CrossRef]

10. Ben-Josef, E.; Moughan, J.; Ajani, J.A.; Flam, M.; Gunderson, L.; Pollock, J.; Myerson, R.; Anne, R.; Rosenthal, S.A.; Willett, C. Impact of Overall Treatment Time on Survival and Local Control in Patients with Anal Cancer: A Pooled Data Analysis of Radiation Therapy Oncology Group Trials 87-04 and 98-11. J. Clin. Oncol. 2010, 28, 5061-5066. [CrossRef]

11. Glynne-Jones, R.; Sebag-Montefiore, D.; Adams, R.; McDonald, A.; Gollins, S.; James, R.; Northover, J.M.; Meadows, H.M.; Jitlal, M. "Mind the Gap"-The Impact of Variations in the Duration of the Treatment Gap and Overall Treatment Time in the First UK Anal Cancer Trial (ACT I). Int. J. Radiat. Oncol. Biol. Phys. 2011, 81, 1488-1494. [CrossRef]

12. Han, K.; Cummings, B.J.; Dawson, L.A.; Ringash, J.; Krzyzanowska, M.K.; Moore, M.J.; Chen, E.X.; Easson, A.M.; Kassam, Z.; Cho, C.; et al. Prospective Evaluation of Acute Toxicity and Quality of Life After IMRT and Concurrent Chemotherapy for Anal Canal and Perianal Cancer. Int. J. Radiat. Oncol. Biol. Phys. 2014, 90, 587-594. [CrossRef]

13. Mitchell, M.P.; Abboud, M.; Eng, C.; Beddar, A.S.; Krishnan, S.; Delclos, M.E.; Crane, C.H.; Das, P. Intensity-modulated Radiation Therapy with Concurrent Chemotherapy for Anal Cancer: Outcomes and toxicity. Am. J. Clin. Oncol. 2014, 37, 461-466. [CrossRef]

14. Call, J.A.; Prendergast, B.M.; Jensen, L.G.; Ord, C.B.; Goodman, K.A.; Jacob, R.; Mell, L.K.; Thomas, C.R.; Jabbour, S.K.; Miller, R.C. Intensity-modulated Radiation Therapy for Anal Cancer: Results from a multi-institutional retrospective cohort study: Results from a multi-institutional retrospective cohort study. Am. J. Clin. Oncol. 2016, 39, 8-12. [CrossRef]

15. Kachnic, L.A.; Winter, K.; Willett, C.G.; Myerson, R.J.; Goodyear, M.D.; Willins, J.; Esthappan, J.; Haddock, M.G.; Rotman, M.; Parikh, P.J.; et al. RTOG 0529: A Phase 2 Evaluation of Dose-Painted Intensity Modulated Radiation Therapy in Combination With 5-Fluorouracil and Mitomycin-C for the Reduction of Acute Morbidity in Carcinoma of the Anal Canal. Int. J. Radiat. Oncol. Biol. Phys. 2013, 86, 27-33. [CrossRef]

16. Yates, A.; Carroll, S.; Kneebone, A.; Tse, R.; Horvath, L.; Byrne, C.; Solomon, M.; Hruby, G. Implementing Intensity-modulated Radiotherapy with Simultaneous Integrated Boost for Anal Cancer: 3 Year Outcomes at Two Sydney Institutions. Clin. Oncol. R. Coll. Radiol. 2015, 27, 700-707. [CrossRef]

17. Mitra, D.; Hong, T.S.; Horick, N.; Rose, B.; Drapek, L.N.; Blaszkowsky, L.S.; Allen, J.N.; Kwak, E.L.; Murphy, J.E.; Clark, J.W.; et al. Long-term outcomes and toxicities of a large cohort of anal cancer patients treated with dose-painted IMRT per RTOG 0529. Adv. Radiat. Oncol. 2017, 2, 110-117. [CrossRef]

18. Arcadipane, F.; Franco, P.; Ceccarelli, M.; Furfaro, G.; Rondi, N.; Trino, E.; Martini, S.; Iorio, G.C.; Mistrangelo, M.; Cassoni, P.; et al. Image-guided IMRT with simultaneous integrated boost as per RTOG 0529 for the treatment of anal cancer. Asia-Pac. J. Clin. Oncol. 2018, 14, 217-223. [CrossRef]

19. Franco, P.; Mistrangelo, M.; Arcadipane, F.; Munoz, F.; Sciacero, P.; Spadi, R.; Migliaccio, F.; Angelini, V.; Bombaci, S.; Rondi, N.; et al. Intensity-Modulated Radiation Therapy with Simultaneous Integrated Boost Combined with Concurrent Chemotherapy for the Treatment of Anal Cancer Patients: 4-Year Results of a Consecutive Case Series. Cancer Investig. 2015, 33, 259-266. [CrossRef]

20. Franco, P.; Arcadipane, F.; Ragona, R.; Mistrangelo, M.; Cassoni, P.; Rondi, N.; Morino, M.; Racca, P.; Ricardi, U. Locally Advanced (T3-T4 or N+) Anal Cancer Treated with Simultaneous Integrated Boost Radiotherapy and Concurrent Chemotherapy. Anticancer Res. 2016, 36, 2027-2032.

21. De Bari, B.; Lestrade, L.; Franzetti-Pellanda, A.; Jumeau, R.; Biggiogero, M.; Kountouri, M.; Matzinger, O.; Miralbell, R.; Bourhis, J.; Ozsahin, M.; et al. Modern intensity-modulated radiotherapy with image guidance allows low toxicity rates and good local control in chemoradiotherapy for anal cancer patients. J. Cancer Res. Clin. Oncol. 2018, 144, 781-789. [CrossRef]

22. Dell'Acqua, V.; Surgo, A.; Arculeo, S.; Zerella, M.A.; Bagnardi, V.; Frassoni, S.; Zampino, M.G.; Ravenda, P.S.; Rotundo, M.S.; Kraja, F.; et al. Intensity-modulated radiotherapy (IMRT) in the treatment of squamous cell anal canal cancer: Acute and early-late toxicity, outcome, and efficacy. Int. J. Color. Dis. 2020, 35, 685-694. [CrossRef]

23. Glynne-Jones, R.; Nilsson, P.; Aschele, C.; Goh, V.; Peiffert, D.; Cervantes, A.; Arnold, D. Anal cancer: ESMO-ESSO-ESTRO clinical practice guidelines for diagnosis, treatment and follow-up. Eur. J. Surg. Oncol. (EJSO) 2014, 40, 1165-1176. [CrossRef] [PubMed]

24. Myerson, R.J.; Garofalo, M.C.; El Naqa, I.; Abrams, R.A.; Apte, A.; Bosch, W.R.; Das, P.; Gunderson, L.L.; Hong, T.S.; Kim, J.J.; et al. Elective Clinical Target Volumes for Conformal Therapy in Anorectal Cancer: A Radiation Therapy Oncology Group Consensus Panel Contouring Atlas. Int. J. Radiat. Oncol. 2009, 74, 824-830. [CrossRef]

25. Ng, M.; Leong, T.; Chander, S.; Chu, J.; Kneebone, A.; Carroll, S.; Wiltshire, K.; Ngan, S.; Kachnic, L. Australasian Gastrointestinal Trials Group (AGITG) Contouring Atlas and Planning Guidelines for Intensity-Modulated Radiotherapy in Anal Cancer. Int. J. Radiat. Oncol. 2012, 83, 1455-1462. [CrossRef] [PubMed]

26. Muirhead, R.; Partridge, M.; Hawkins, M.A. A tumor control probability model for anal squamous cell carcinoma. Radiother. Oncol. 2015, 116, 192-196. [CrossRef] [PubMed]

27. John, M.; Pajak, T.; Flam, M.; Hoffman, J.; Markoe, A.; Wolkov, H.; Paris, K. Dose escalation in chemoradiation for anal cancer: Preliminary results of RTOG 92-08. Cancer J. Sci. Am. 1996, 2, 205-211. 
28. Arcadipane, F.; Silvetti, P.; Olivero, F.; Gastino, A.; De Luca, V.; Mistrangelo, M.; Cassoni, P.; Racca, P.; Gallio, E.; Lesca, A.; et al. Bone Marrow-Sparing IMRT in Anal Cancer Patients Undergoing Concurrent Chemo-Radiation: Results of the First Phase of a Prospective Phase II Trial. Cancers 2020, 12, 3306. [CrossRef] [PubMed]

29. Kim, J.J.; Tannock, I.F. Repopulation of cancer cells during therapy: An important cause of treatment failure. Nat. Rev. Cancer 2005, 5, 516-525. [CrossRef] [PubMed]

30. Muirhead, R.; Adams, R.A.; Gilbert, D.C.; Harrison, M.; Glynne-Jones, R.; Sebag-Montefiore, D.; Hawkins, M. National Guidance for IMRT in Anal Cancer. 2016. Available online: http: / / analimrtguidance.co.uk/National-Guidance-IMRT-Anal-Cancer-V4 -Jan17.pdf (accessed on 3 October 2018).

31. Shakir, R.; Adams, R.; Cooper, R.; Downing, A.; Geh, I.; Gilbert, D.; Jacobs, C.; Jones, C.; Lorimer, C.; Namelo, W.C.; et al. Patterns and Predictors of Relapse Following Radical Chemoradiation Therapy Delivered Using Intensity Modulated Radiation Therapy With a Simultaneous Integrated Boost in Anal Squamous Cell Carcinoma. Int. J. Radiat. Oncol. 2020, 106, 329-339. [CrossRef]

32. Sekhar, H.; Zwahlen, M.; Trelle, S.; Malcomson, L.; Kochhar, R.; Saunders, M.P.; Sperrin, M.; van Herk, M.; Sebag-Montefiore, D.; Egger, M.; et al. Nodal stage migration and prognosis in anal cancer: A systematic review, meta-regression, and simulation study. Lancet Oncol. 2017, 18, 1348-1359. [CrossRef]

33. Cancer Therapy Evaluation Program (CTEP) of the National Cancer Institute (NCI). Common Terminology Criteria for Adverse Events (CTCAE); Version 4.0; v4.02; US Department of Health and Human Services, National Institutes of Health, National Cancer Institute, Bethesda: Rockville, MD, USA, 2009.

34. Eisenhauer, E.A.; Therasse, P.; Bogaerts, J.; Schwartz, L.H.; Sargent, D.; Ford, R.; Dancey, J.; Arbuck, S.; Gwyther, S.; Mooney, M.; et al. New response evaluation criteria in solid tumours: Revised RECIST guideline (version 1.1). Eur. J. Cancer 2009, 45, $228-247$. [CrossRef]

35. Glynne-Jones, R.; Adams, R.; Lopes, A.; Meadows, H. Clinical endpoints in trials of chemoradiation for patients with anal cancer. Lancet Oncol. 2017, 18, e218-e227. [CrossRef]

36. Fish, R.; Sanders, C.; Ryan, N.; Van Der Veer, S.; Renehan, A.G.; Williamson, P.R. Systematic review of outcome measures following chemoradiotherapy for the treatment of anal cancer (CORMAC). Colorectal Dis. 2018, 20, 371-382. [CrossRef] [PubMed] 